\title{
Impaired folate 1-carbon metabolism causes formate-preventable hydrocephalus in glycine decarboxylase-deficient mice
}

\author{
Chloe Santos, ${ }^{1}$ Yun Jin Pai, ${ }^{1}$ M. Raasib Mahmood, ${ }^{1}$ Kit-Yi Leung, ${ }^{1}$ Dawn Savery, ${ }^{1}$ Simon N. Waddington, ${ }^{2,3}$ Andrew J. Copp, ${ }^{1}$ \\ and Nicholas D.E. Greene ${ }^{1}$
}

'UCL Great Ormond Street Institute of Child Health and ${ }^{2}$ EGA Institute for Women's Health, University College London, London, United Kingdom. ${ }^{3}$ MRC Antiviral Gene Therapy Research Unit, Faculty of Health Science, University of the Witswatersrand, Johannesburg, South Africa.

\begin{abstract}
Ventriculomegaly and hydrocephalus are associated with loss of function of glycine decarboxylase (Cldc) in mice and in humans suffering from non-ketotic hyperglycinemia (NKH), a neurometabolic disorder characterized by accumulation of excess glycine. Here, we showed that ventriculomegaly in Gldc-deficient mice is preceded by stenosis of the Sylvian aqueduct and malformation or absence of the subcommissural organ and pineal gland. Gldc functions in the glycine cleavage system, a mitochondrial component of folate metabolism, whose malfunction results in accumulation of glycine and diminished supply of glycine-derived 1-carbon units to the folate cycle. We showed that inadequate 1-carbon supply, as opposed to excess glycine, is the cause of hydrocephalus associated with loss of function of the glycine cleavage system. Maternal supplementation with formate prevented both ventriculomegaly, as assessed at prenatal stages, and postnatal development of hydrocephalus in Cldc-deficient mice. Furthermore, ventriculomegaly was rescued by genetic ablation of 5,10-methylene tetrahydrofolate reductase (Mthfr), which results in retention of 1-carbon groups in the folate cycle at the expense of transfer to the methylation cycle. In conclusion, a defect in folate metabolism can lead to prenatal aqueduct stenosis and resultant hydrocephalus. These defects are preventable by maternal supplementation with formate, which acts as a 1-carbon donor.
\end{abstract}

\section{Introduction}

Hydrocephalus results from abnormal cerebrospinal fluid (CSF) hydrodynamics (overproduction, diminished drainage, or impaired flow) leading to progressive enlargement of the cerebral ventricular system and subsequent pathology (1). Hydrocephalus can be acquired (following injury, infection, tumor formation, or trauma) or arise as a congenital condition, in isolation or associated with structural abnormalities such as Dandy-Walker or Chiari II malformations $(2,3)$. Although common (affecting $0.5-1$ per 1,000 live births), the molecular pathophysiology of isolated congenital hydrocephalus is known in only a relatively small proportion of cases, owing to heterogeneity and multifactorial etiology (4-6).

A potential causal effect of impaired function of the glycine cleavage system (GCS) is highlighted by occurrence of hydrocephalus in association with non-ketotic hyperglycinemia $(\mathrm{NKH})$, a life-limiting autosomal recessive neurometabolic disorder characterized by accumulation of glycine in body fluids and tissues $(7$, 8). NKH results from mutation of GCS-encoding genes, with the majority of patients carrying mutations in GLDC (glycine decar-

Authorship note: CS and YJP contributed equally to this work. Conflict of interest: The authors have declared that no conflict of interest exists. Copyright: $\odot 2020$ Santos et al. This is an open access article published under the terms of the Creative Commons Attribution 4.0 International License.

Submitted: August 5, 2019; Accepted: November 20, 2019; Published: February 4, 2020. Reference information: / Clin Invest. 2020;130(3):1446-1452

https://doi.org/10.1172/JCI132360. boxylase) $(9,10)$. Hydrocephalus arises in approximately $8 \%$ of $\mathrm{NKH}$ patients and enlarged ventricles are commonly found on imaging (15 of 41 patients in one clinical survey) $(8,11)$.

A direct link between GLDC loss of function and hydrocephalus was confirmed by analysis of mice carrying hypomorphic $\left(G l d c^{G T 1}\right)$ or null $\left(G l d c^{G T 2}\right)$ alleles of the murine homolog, Gldc. A proportion of Gldc mutants die perinatally, owing to neural tube defects (NTDs) resulting from failed neural tube closure $(12,13)$. However, among Gldc-deficient mice that survive postnatally, hydrocephalus becomes evident by 5-7 weeks of age in $20 \%-25 \%$ of homozygotes ( $G l d c^{G T 1 / G T 1}$ ), with a characteristic domed head, distorted cranium, and severely enlarged lateral ventricles (12). These mice also show signs of $\mathrm{NKH}$, including elevated glycine concentration in body fluids and tissues and premature lethality (12).

A prenatal origin of hydrocephalus was demonstrated by histological analysis of litters at embryonic day 18.5 (E18.5), which revealed enlarged lateral ventricles in Gldc-deficient (Gldc $c^{G T 1 / G T 1}$ or $\left.G l d c^{G T 1 / G T 2}\right)$ mice $(12,14)$. This is consistent with the association of ventriculomegaly, enlargement of the cerebral ventricles, with postnatal hydrocephalus in humans (15). Serial imaging of individual Gldc $c^{G T 1 / G T 2}$ fetuses at successive gestational time points by in utero high-frequency ultrasound showed onset of ventriculomegaly between E16.5 and E18.5 (14).

The physiological (communicating vs. noncommunicating) and metabolic mechanisms underlying GLDC-related hydrocephalus have not been determined. The GCS is a mitochondrial enzyme complex that mediates decarboxylation of glycine, 

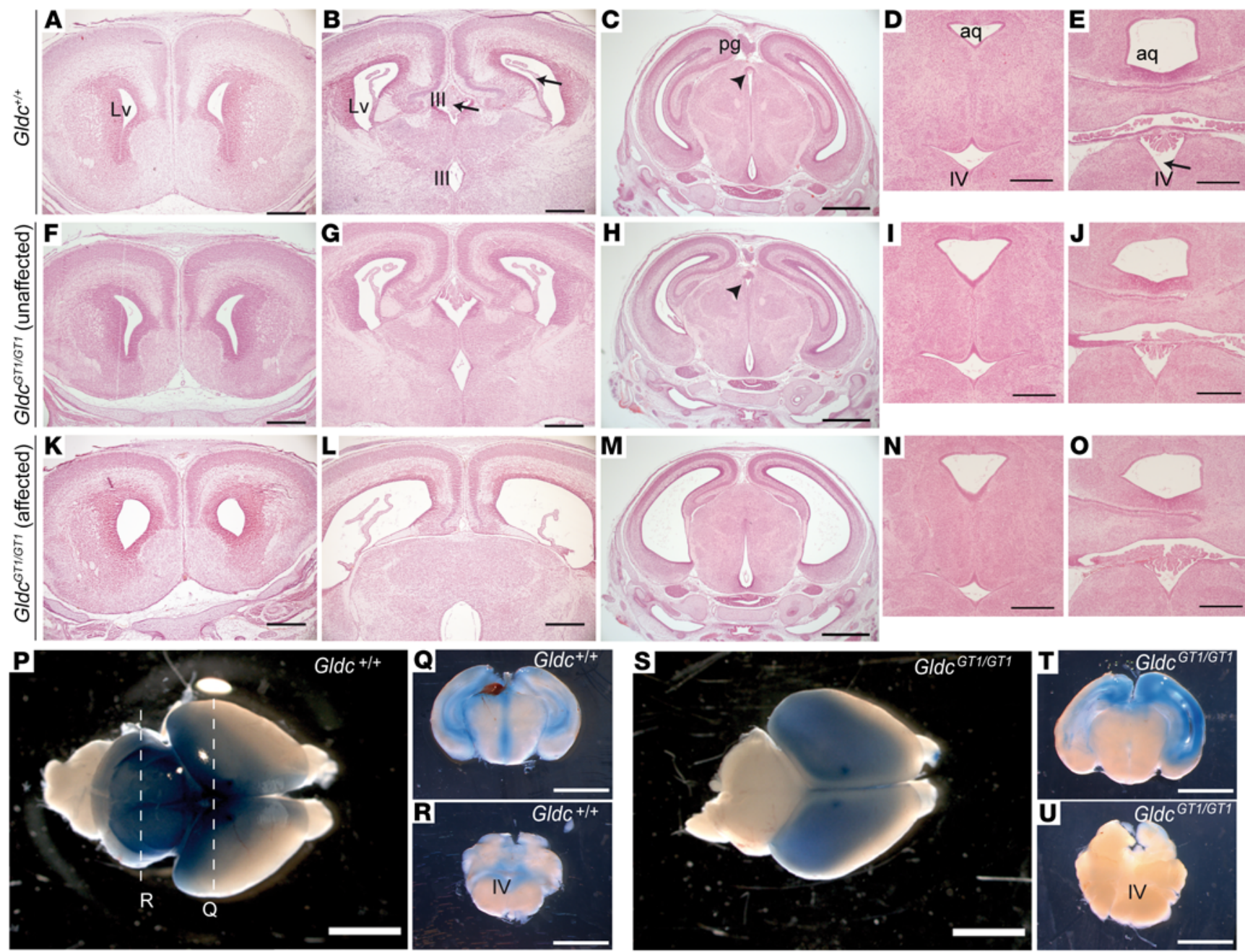

Figure 1. GIdc deficiency results in ventriculomegaly. Unlike wild-type (A-E) and unaffected Gldc ${ }^{G T / G T 1}$ (F-J) fetuses, a subset (7 of 13) of Gldc ${ }^{G T 1 / G T 1}$ fetuses (K-0) were affected by enlargement of the lateral (LV) and third (III) ventricles at E18.5. The fourth ventricle (IV, arrow in E) does not differ in size between genotypes (compare $\mathbf{D}$ with $\mathbf{E}$, I with $\mathbf{~ J , ~ a n d ~} \mathbf{N}$ with $\mathbf{0}$ ), nor does the aqueduct (aq) at this posterior axial level. Choroid plexus is detected in lateral, third, and fourth ventricles (arrows in $\mathbf{B}$ ) of all genotypes. However, the pineal gland (pg) and subcommissural organ (arrowheads in $\mathbf{C}$ and $\mathbf{H}$ ) are absent in $G / d c^{C T 1 / G T 1}$ fetuses displaying ventriculomegaly (M). (P-U) Following bilateral injection into the lateral ventricles of neonatal mice (P, Q, S, and $\mathbf{T}$ ), dye distributed throughout the ventricular system including the fourth ventricle (IV) of Gldc ${ }^{+/+}$(R) but not Gldc $c^{\mathrm{GT} / \mathrm{GT1}}$ (U) mice. Scale bars: 1 mm (C, H, and $\mathbf{M}), 5 \mathrm{~mm}(\mathbf{P}-\mathbf{U})$, and $0.5 \mathrm{~mm}$ (other panels).

with concomitant transfer of a 1-carbon group to tetrahydrofolate (THF), generating 5,10-methylene THF in folate 1-carbon metabolism (FOCM) (9). Hence, GCS loss of function not only causes accumulation of glycine, but also prevents transfer of glycinederived 1-carbon units to FOCM (13). Excess glycine is thought to lead to neurological features of NKH, such as epilepsy $(8,9)$, whereas NTDs result from impaired FOCM (13). Potential mechanisms underlying the development of ventriculomegaly and hydrocephalus could therefore include consequences of FOCM suppression or glycine accumulation in CSF or neural tissues. For example, it is proposed that altered osmolality of CSF (a potential effect of excess glycine) could result in net movement of water into the ventricles, leading to abnormal CSF hydrodynamics (16). Alternatively, FOCM is required for provision of 1-carbon groups for key cellular processes including nucleotide biosynthesis and methylation reactions, whose disturbance could plausibly lead to ventriculomegaly. Here, we investigated the cause of hydrocephalus and the requirement for FOCM in ventricular development in Gldc-deficient mice.

\section{Results and Discussion}

In our previous ultrasound analysis of litters at E18.5, Gldc-deficient $\left(G l d c^{G T 1 / G T 2}\right.$ ) fetuses that exhibited enlarged lateral ventricles did not differ from wild types in volume of the fourth ventricle or cerebellum (14). Similarly, we found that among $G l d c^{G T / G T 1}$ fetuses with obvious dilatation of the lateral and third ventricles ( 7 of 13 Gld $\left.c^{G T / G T 1}\right)$, more posterior structures including the fourth ventricle appeared comparable to wild types (Figure 1).

Specific expansion of the ventricular system rostral to the aqueduct of Silvius indicated the possibility of mechanical obstruction. Consistent with this hypothesis, after injection into the lateral ventricles, aqueous dye circulated to the posterior aqueduct and fourth 


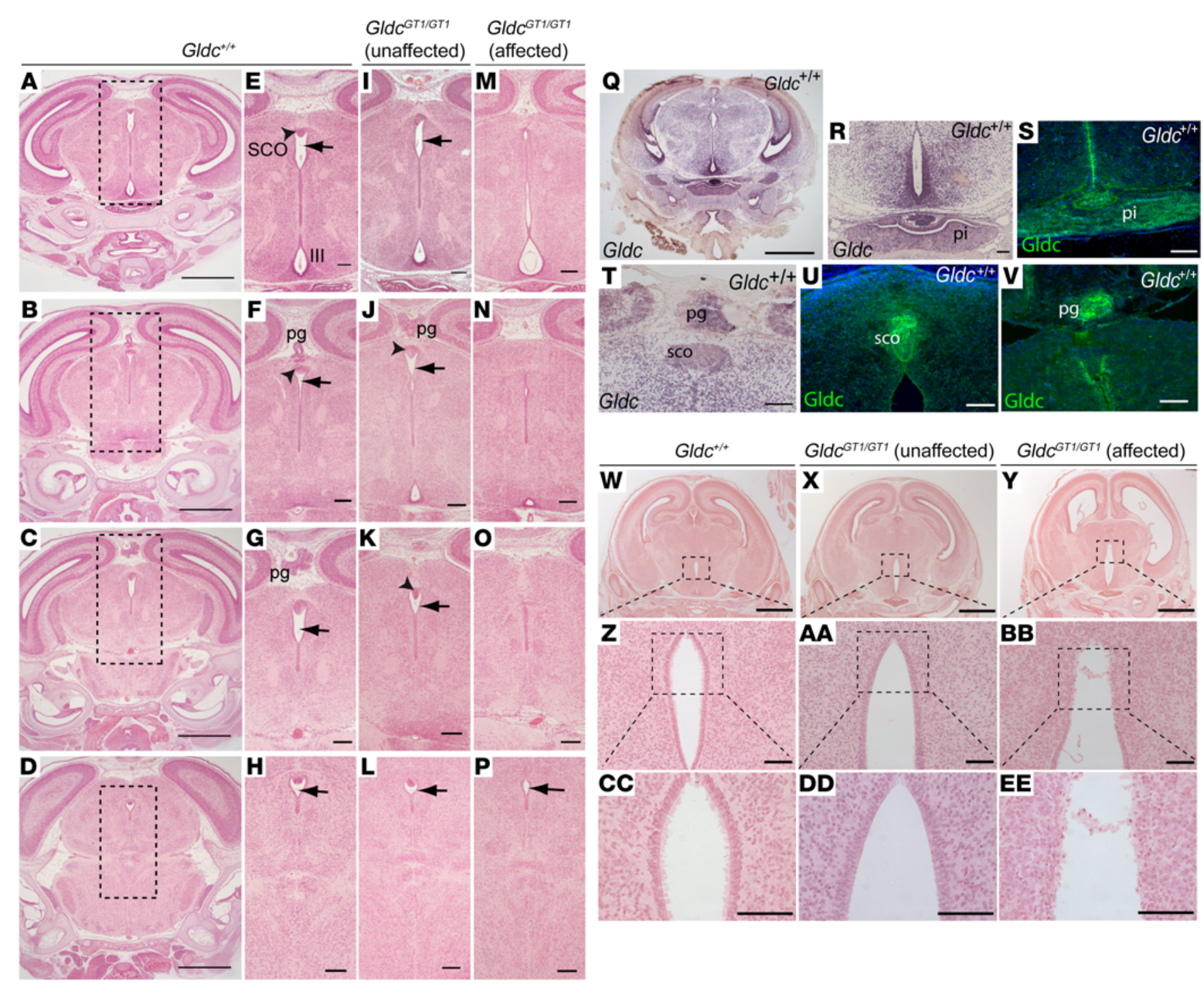

Figure 2. Ventriculomegaly is associated with aqueduct stenosis in Gldc-deficient fetuses. Coronal sections in a rostral-caudal sequence (at levels shown in A-D in wild-type brain) show continuity of the aqueduct of Sylvius in Gldc c/+ (arrows in E-H) and unaffected Gldc $c^{C T 1 / G T 1}$ (I-L) fetuses at E18.5. In contrast, the aqueduct narrows and exhibits discontinuities in Gld $c^{G T / G T 1}$ mutants with ventriculomegaly (affected) (M-P). Boxed areas in A-D show enlarged regions in E-P. At E16.5, Gldc mRNA is widely expressed in the brain (Q, R, and $\mathbf{T}$ ), with abundant expression in the pineal gland (pg), subcommissural organ (sco), and pituitary (pi). Immunohistochemistry confirms localization of Gldc protein at these sites at E18.5 (S, U, and $\mathbf{V})$. The ependymal cell lining of the third ventricle (boxed in $\mathbf{W}-\mathbf{Y}$, enlarged in Z-EE) appears disrupted in Gldc ${ }^{G T 1 / G T 1}$ fetuses at E18.5 (Y). Scale bars: $0.1 \mathrm{~mm}$ (R-V and Z-EE), $0.5 \mathrm{~mm}(\mathbf{A}-\mathbf{P})$, and $1 \mathrm{~mm}(\mathbf{Q}$ and $\mathbf{W}-\mathbf{Y})$.

ventricles in wild-type and $G l d c^{G T T /+}$ neonatal mice but not in some Gldc GT1/GT1 littermates (Figure 1, P-U and Supplemental Figure 1; supplemental material available online with this article; https:// doi.org/10.1172/JCI132360DS1). Among wild-type and unaffected $G l d c^{G T 1 / G T 1}$ fetuses at E18.5, the aqueduct lumen was continuous and could be followed in serial sections from the third to fourth ventricles (Figure 2). In contrast, the aqueduct was very narrow or completely occluded in all $G l d c^{G T 1 / G T 1}$ fetuses that displayed ventriculomegaly (Figure 2, M-P, and Supplemental Figure 2).

At E18.5, the subcommissural organ (SCO) and pineal gland are visible in the roof of the aqueduct in wild-type and unaffected Gldc-deficient fetuses (Figure 2, A-L). However, in all the affected Gldc-deficient fetuses the SCO was absent and the pineal gland was absent or malformed (Figure 1M and Figure 2, M-P). SCO agenesis is associated with development of both communicating and noncommunicating forms of hydrocephalus in mice, as seen in genetic mutants for Msx1, Pax6, and Rfx3 or overexpression of Sox3 $(17,18)$. A hypoplastic pineal gland is also observed with postnatal hydrocephalus in $L h x 9$-null mice (19).

Gldc is expressed throughout the neuroepithelium at E9.5 (12). At developmental stages when ventriculomegaly arose, we found widespread expression of Gldc in the brain at E16.5, including the pineal gland, SCO, and pituitary gland (Figure 2, Q, T, and R). Immunostaining confirmed the presence of Gldc protein in these structures at E18.5 (Figure 2, S, U, and V).

Hydrocephalus has been associated with denudation of the ependymal cell layer lining the ventricles and subsequent occlusion of the aqueduct $(17,20)$. Lack or abnormal function of 

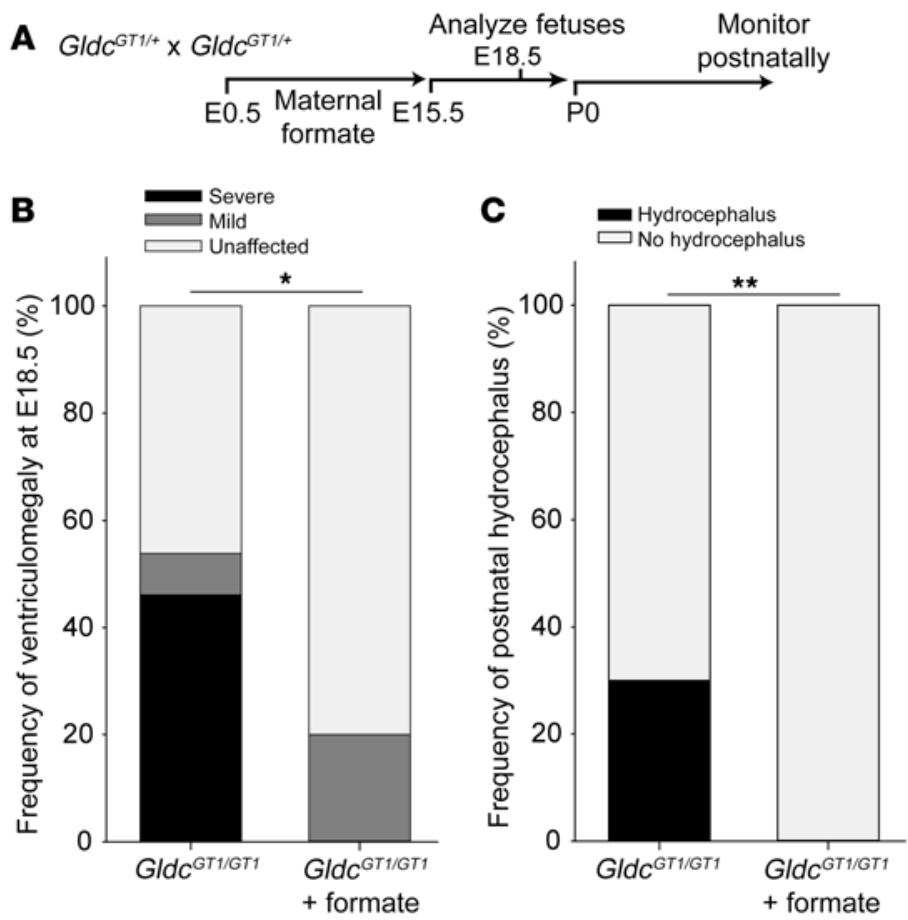
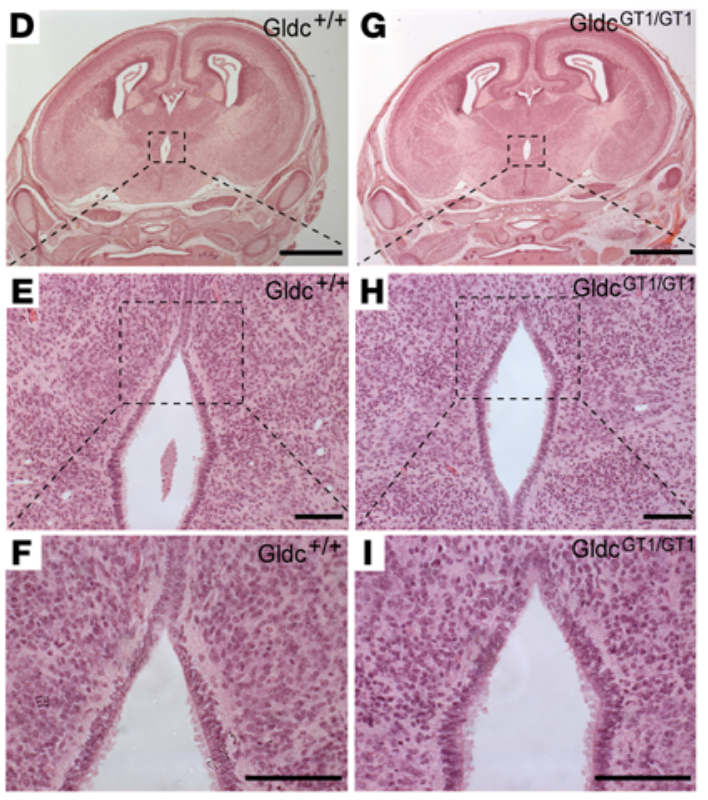

Figure 3. Maternal formate supplementation prevents ventriculomegaly and hydrocephalus. (A) GIdc $c^{G T 1 /+}$ mice were mated and pregnant females were supplemented with formate from E0.5 to E15.5. (B) At E18.5, there was a significantly greater proportion of Gldc ${ }^{G T 1 / G T 1}$ fetuses that were unaffected ( $n$ $=8$ of 10) in formate-treated litters than among Gldc $c^{G T 1 / G T 1}$ fetuses ( $n=6$ of 13) in control litters. ${ }^{*} P<0.05$ by Fisher's exact test. (C) Among offspring of formate-supplemented mice that were monitored at postnatal stages, none of the Gldc ${ }^{\text {GT1/GT1 }}$ mice ( $n=0$ of 35 ) developed hydrocephalus, whereas $30 \%$ (6 of 20) of nonsupplemented GId $c^{G T 1 / G T 1}$ offspring developed hydrocephalus by $6-7$ weeks. ${ }^{* *} P<0.002$ by Fisher's exact test. Number of litters: $n=19$ supplemented and 35 nonsupplemented. (D-I) The ependymal lining of the third ventricle appeared intact in formate-treated fetuses at E18.5. Scale bars: $1 \mathrm{~mm}$ (D and $\mathbf{G}$ ) and $100 \mu \mathrm{m}$ (other panels).

ependymal motile cilia may also result in hydrocephalus without aqueduct stenosis, possibly owing to impaired CSF flow $(17,20)$. We found that the ependymal cell layer was intact in the ventricular system of wild-type $(n=5)$ and unaffected $G l d c^{G T 1 / G T 1}(n$ = 4) fetuses at E18.5 (Figure 2, W, X, Z, AA, CC, and DD, and Supplemental Figure 2). Abnormalities were not observed in the ependymal cell layer of the lateral ventricles in $G l d c^{G T 1 / G T 1}$ fetuses with ventriculomegaly (Supplemental Figure 2, G-L), but this layer appeared disrupted in the dorsal region of the third ventricle in most (4 out of 5; Figure 2, Y, BB, and EE) but not all (Supplemental Figure 3) fetuses. The fetal onset of ventriculomegaly, prior to maturation of multiciliated ependyma (in the first postnatal week in mice), and largely intact ependyma suggest that disruption of this cell layer is unlikely to be causal. Together, the presence of enlarged lateral and third ventricles, unaffected fourth ventricle, impaired dye distribution, and late-fetal onset of ventriculomegaly implicate aqueduct stenosis as the structural abnormality causing a noncommunicating form of hydrocephalus in Gldc-deficient fetuses.

We next investigated the metabolic basis of hydrocephalus in Gldc-deficient mice. Loss of function of GCS causes accumulation of glycine, with significantly elevated levels in Gldc-deficient embryos by E11.5 (12). Metabolic labeling shows that the contribution of glycine-derived 1-carbon units to FOCM is also ablated in Gldc-deficient embryos, with consequent alterations in the relative abundance of folates $(12,13)$.
We found that exogenous supply of 1-carbon units by maternal formate supplementation normalized the folate profile in Gldcdeficient embryos and prevented NTDs, despite the tissue glycine concentration remaining elevated $(12,13)$. Based on this strategy, pregnant dams were supplemented with formate prior to collection of litters for evaluation of ventriculomegaly (Figure 3A). Histological examination divided $G l d c^{G T 1 / G T 1}$ fetuses into 2 main categories: a group that exhibited severely dilated lateral ventricles with absent SCO and pineal gland (Figure 1M) and another with normal appearance, indistinguishable from wild type (Figure $1 \mathrm{H}$ and Supplemental Figure 4). A few fetuses displayed an intermediate phenotype in which the SCO or pineal gland was detectable but appeared abnormal, accompanied by mild ventricle dilation (Supplemental Figure 4). Formate supplementation led to significant normalization of development (Figure 3B), with none of the treated Gldc $c^{G T 1 / G T 1}$ fetuses displaying severe ventriculomegaly or absence of the SCO and pineal gland. Similarly, the ependymal cell layer was intact in all formate-treated fetuses examined $(n=6$ unaffected and 1 intermediate; Figure 3, D-I).

Having found that formate supplementation could prevent fetal ventriculomegaly, we asked whether this prenatal treatment rescued postnatal onset of hydrocephalus. Gld $c^{G T 1 /+}$ dams were supplemented with formate for the first 15 days of pregnancy and offspring were monitored for development of abnormalities until 6-7 weeks of age. Notably, none of the Gld $c^{G T 1 / G T 1}$ offspring $(n=35)$ of formate-supplemented mice developed hydrocephalus, unlike 
A

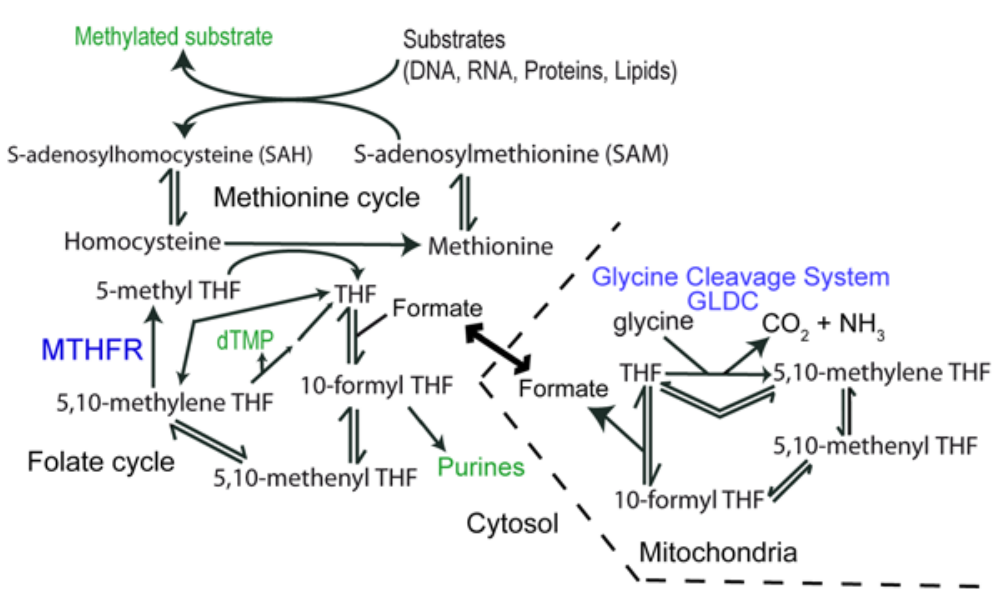

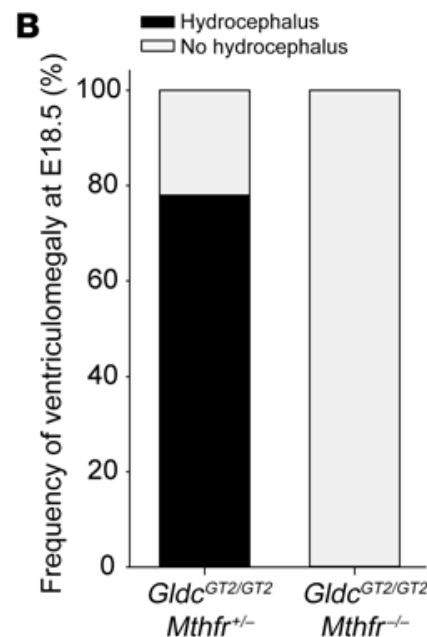
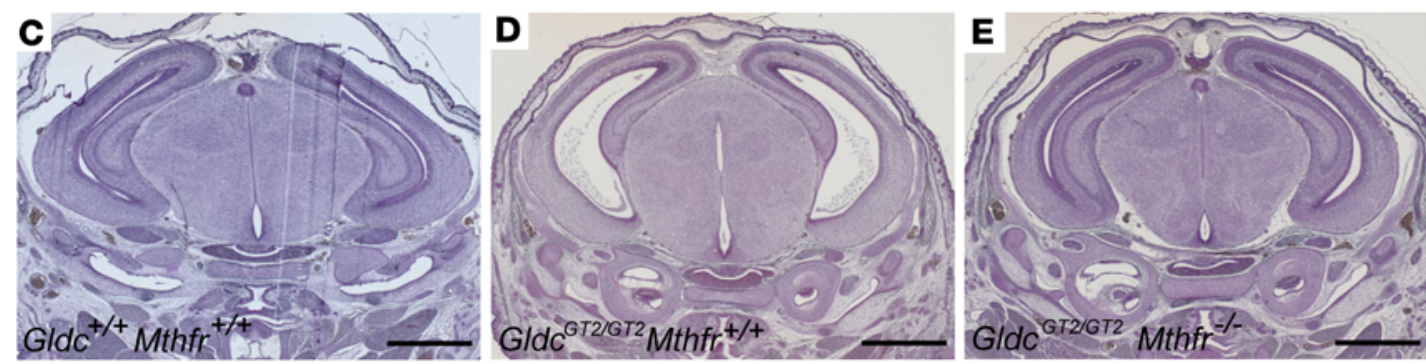

Figure 4. Mthfr deletion in Gldc-deficient mice normalizes development of the aqueduct, subcommisural organ, and pineal gland. (A) Outline of folate and methionine cycles, with key outputs in green text and relevant enzymes in blue text. Lack of Mthfr activity prevents transfer of 1-carbon units from 5,10-methy-

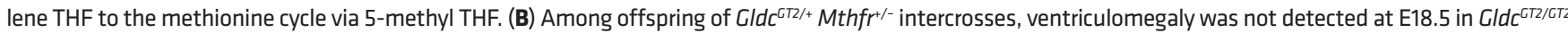
Mthfr ${ }^{1-}$ fetuses $(n=5)$, but occurred at high frequency among Gldc CT2/GT2 fetuses that were heterozygous $(n=7)$ or wild type $(n=2)$ for Mthfr $(P<0.025$ by Fisher's exact test). (C-E) Sections of E15.5 wild-type (C), Gld $c^{G T 2 / G T 2}(\mathbf{D})$, and Gld $c^{G T 2 / G T 2} \mathrm{Mthfr}^{\prime-}$ (E) brains showing that absence of Mthfr restores the wild-type appearance of the pineal gland, subcommissural organ, and aqueduct, and prevents ventriculomegaly, in Cldc-null fetuses. Scale bars: $1 \mathrm{~mm}$.

nonsupplemented $G l d c^{G T 1 / G T 1}$ in which hydrocephalus arose in $30 \%$ of mice (Figure $3 C$ ), similar to previous studies $(12,14)$.

Prevention of ventriculomegaly/hydrocephalus by prenatal formate supplementation implicates insufficient supply of 1-carbon groups to FOCM as the underlying causative mechanism. The folate cycle intermediates, 5,10-methylene tetrahydrofolate (THF) and 10-formyl THF, act as 1-carbon donors in biosynthesis of thymidylate and purines. Alternatively, 1-carbon groups can be transferred to the methionine cycle via donation of a methyl group from 5-methyl THF to homocysteine (Figure 4A). To ask whether ventriculomegaly derives from suppression of the folate cycle or inadequate supply of 1-carbon groups to the methionine cycle we bred Gldc-deficient fetuses that lack 5,10-methylene tetrahydrofolate reductase (Mthfr). Compound null $\mathrm{Gld}^{G T 2 / G T 2} \mathrm{Mthfr}^{-1-}$ embryos cannot generate 5-methyl THF and 1-carbon groups are therefore retained in the folate cycle (13).

Analysis of litters produced by intercross of compound heterozygous, Gld $c^{G T 2 /+} M t h f r^{+/-}$mice revealed venticulomegaly in Gld $c^{G T 2 / G T 2}$ fetuses that were wild type or heterozygous at the Mthfr locus (Figure 4). In contrast, all Gldc/Mthfr double-knockout fetuses lacked ventriculomegaly, demonstrating a significant protective effect of the Mthfr null mutation. This preventive effect highlights the folate cycle, rather than methionine cycle, as the subset of FOCM reactions that are critical in prevention of aque- duct stenosis. Given the key role of the folate cycle in providing precursors for thymidylate and purine synthesis we speculate that defects result from impaired nucleotide biosynthesis in the neuroepithelium. The next step in this research will be to further refine the developmental stage and tissue site(s) in which folate cycle disruption subsequently leads to malformations of the SCO, pineal gland, and aqueduct.

In animal models, a potential link between folate status and hydrocephalus was noted in dietary studies examining nutritional effects of folate and vitamin $B_{12}(21,22)$. Some neonatal offspring of rats fed synthetic diets lacking folic acid and vitamin $B_{12}$ exhibited enlarged ventricles attributed to possible blockage of the cerebral aqueduct (21). However, speculation remained over the relative importance of folic acid and vitamin $\mathrm{B}_{12}$ and whether FOCM was impaired in these models (22).

Abnormal folate transport to the brain has been proposed to contribute to aqueduct obstruction in the hydrocephalic Texas (H-Tx) rat $(23,24)$. Interestingly, however, hydrocephalus is not commonly described among neurological signs associated with the very low levels of folate in CSF that occur in hereditary folate malabsorption, caused by mutations in SLC46A1 (proton-coupled folate transporter; PCFT) or cerebral folate deficiency, caused by mutations in FOLR1 (folate receptor $\alpha$; FR $\alpha$ ) (25-27). On the other hand, hydrocephalus can arise in patients with severe MTHFR 
deficiency or remethylation disorders (including cblC disease) (28). MTHFR-related hydrocephalus may be of the communicating form (29), and it is not clear to what extent aqueduct stenosis also contributes. Nevertheless, impaired methionine synthase activity (as in cblC patients) could lead to accumulation of 5-methyl THF and thereby deplete 1-carbon units from the folate cycle (methyl trap). This suggests the potential for a shared biochemical mechanism underlying hydrocephalus caused by methionine synthase or Gldc deficiency.

Here, we found that impaired enzymatic activity within mitochondrial FOCM, as opposed to diminished exogenous supply or a methyl trap, can be a direct cause of aqueduct stenosis, the most common known cause of congenital hydrocephalus in humans. The presence of hydrocephalus and/or enlarged ventricles in NKH patients and in Gldc-deficient mice suggests that the requirements for GCS activity are shared. Mutations in GLDC may also predispose to failed neurulation in humans as in mice, missense variants having been identified in some patients with NTDs (30-32), including functional mutations found in both NTDs and NKH $(30,31)$.

Further evidence for a molecular link between congenital hydrocephalus and NTDs has come from identification of putative causal mutations in TRIM71, SMARCC1, and PTCH1 in patients with communicating and obstructive forms of hydrocephalus (6). Loss of function of each of these genes in mice causes cranial NTDs (33-35). Although the association of hydrocephalus with spina bifida is usually considered a secondary manifestation, one could speculate that they may be independent malformations with a shared genetic origin in some individuals. Hence, although NTDs and hydrocephalus arise at different developmental stages, the potential for prevention of both defects by formate supplementation suggests that there may be a related cellular mechanism, serving as a starting point for further research toward understanding the causation, pathogenesis, and primary prevention of these related conditions.

\section{Methods}

Further details are provided in the supplemental material.

Gldc-deficient mice carried gene-trap alleles denoted Gldc ${ }^{G T 1}(12)$ or $G l d c^{G T 2}$ (13). Mthfr-null mice were previously described (36). Litters were generated by overnight matings, with the following day designated E0.5. Injection of the lateral ventricles was performed at P1. Mice were genotyped by PCR of genomic DNA $(12,13)$. Sodium formate $(30$ $\mathrm{mg} / \mathrm{mL}$ ) was added to drinking water $(12,13)$ from E0.5 to E15.5 and dams were then returned to normal drinking water.

Bouin's fixed, paraffin-embedded samples were sectioned $(8 \mu \mathrm{m})$ and stained with hematoxylin and eosin. In situ hybridization was performed on sections using a digoxygenin-labeled antisense probe for Gldc (12). Immunostaining was performed using anti-Gldc (1:300; Atlas Antibodies, HPA002318) with Invitrogen anti-rabbit Alexa Fluor secondary (Thermo Fisher Scientific, A11034) antibodies.

Study approval. Studies were carried out under regulations of the Animals (Scientific Procedures) Act 1986 of the United Kingdom (UK) Government and approved by the UCL Animal Welfare and Ethical Review Body, London, UK.

Statistics. Statistical analysis was performed by Fisher's exact test, with $P$ less than 0.05 considered significant.

\section{Author contributions}

NDEG, AJC, and KYL designed the study. CS, YJP, MRM, KYL, DS, and SNW conducted the investigations. NDEG wrote the manuscript, which was edited by NDEG and AJC.

\section{Acknowledgments}

The authors are grateful for assistance from Diana Gold Diaz and Sandra Castro, and Rima Rozen for supply of Mthfr mice. Studies were funded by the Medical Research Council (N003713), Newlife the Charity for Disabled Children, Action Medical Research (GN2403), and Joseph's Goal. YJP was supported by the Public Service Department of Malaysia. This work was supported by the NIHR Great Ormond Street Hospital Biomedical Research Centre.

Address correspondence to: Nicholas D.E. Greene, Developmental Biology \& Cancer Research and Teaching Department, UCL Great Ormond Street Institute of Child Health, 30 Guilford Street, London, WC1N 1EH, United Kingdom. Phone: 44.2079052217; Email:n.greene@ucl.ac.uk.

YJP's present address is: RIKEN Center for Brain Science, Wako, Saitama, Japan.
1. Rekate HL. A contemporary definition and classification of hydrocephalus. Semin Pediatr Neurol. 2009;16(1):9-15.

2. McAllister JP. Pathophysiology of congenital and neonatal hydrocephalus. Semin Fetal Neonatal Med. 2012;17(5):285-294.

3. Kousi M, Katsanis N. The genetic basis of hydrocephalus. Annu Rev Neurosci. 2016;39:409-435.

4. Tully HM, Dobyns WB. Infantile hydrocephalus: a review of epidemiology, classification and causes. Eur JMed Genet. 2014;57(8):359-368.

5. Dewan MC, et al. Global hydrocephalus epidemiology and incidence: systematic review and meta-analysis. J Neurosurg. 2018;130(4):1-15.

6. Furey CG, et al. De novo mutation in genes regulating neural stem cell fate in human congenital hydrocephalus. Neuron. 2018;99(2):302-314.e4.

7. Van Hove JL, et al. Acute hydrocephalus in nonketotic hyperglycinemia. Neurology. 2000;54(3):754-756.

8. Hennermann JB, Berger JM, Grieben U, Scharer G, Van Hove JL. Prediction of long-term outcome in glycine encephalopathy: a clinical survey. J Inherit Metab Dis. 2012;35(2):253-261.

9. Kikuchi G, Motokawa Y, Yoshida T, Hiraga K. Glycine cleavage system: reaction mechanism, physiological significance, and hyperglycinemia. Proc Jpn Acad, Ser B, Phys Biol Sci. 2008;84(7):246-263.

10. Coughlin CR, et al. The genetic basis of classic nonketotic hyperglycinemia due to mutations in GLDC and AMT. Genet Med. 2017;19(1):104-111.

11. Hoover-Fong JE, Shah S, Van Hove JL, Applegarth D, Toone J, Hamosh A. Natural history of nonketotic hyperglycinemia in 65 patients. Neurology. 2004;63(10):1847-1853.
12. Pai YJ, et al. Glycine decarboxylase deficiency causes neural tube defects and features of non-ketotic hyperglycinemia in mice. Nat Commun. 2015;6:6388.

13. Leung KY, et al. Partitioning of one-carbon units in folate and methionine metabolism is essential for neural tube closure. Cell Rep. 2017;21(7):1795-1808.

14. Autuori MC, et al. Use of high-frequency ultrasound to study the prenatal development of cranial neural tube defects and hydrocephalus in Gldc-deficient mice. Prenat Diagn. 2017;37(3):273-281.

15. Pisapia JM, Sinha S, Zarnow DM, Johnson MP, Heuer GG. Fetal ventriculomegaly: Diagnosis, treatment, and future directions. Childs Nerv Syst. 2017;33(7):1113-1123.

16. Orešković D, Radoš M, Klarica M. New concepts of cerebrospinal fluid physiology and devel- 
opment of hydrocephalus. Pediatr Neurosurg. 2017;52(6):417-425.

17. Huh MS, Todd MA, Picketts DJ. SCO-ping out the mechanisms underlying the etiology of hydrocephalus. Physiology (Bethesda). 2009;24:117-126.

18. Lee $\mathrm{K}$, et al. Congenital hydrocephalus and abnormal subcommissural organ development in Sox 3 transgenic mice. PLoS One. 2012;7(1):e29041.

19. Yamazaki F, et al. The Lhx9 homeobox gene controls pineal gland development and prevents postnatal hydrocephalus. Brain Struct Funct. 2015;220(3):1497-1509.

20. Del Bigio MR. Ependymal cells: biology and pathology. Acta Neuropathol. 2010;119(1):55-73.

21. Overholser MD, Whitley JR, O'Dell BL, Hogan AG. The ventricular system in hydrocephalic rat brains produced by a deficiency of vitamin B12 or of folic acid in the maternal diet. Anat Rec. 1954;120(4):917-933.

22. Woodard JC, Newberne PM. Relation of vitamin B12 and one-carbon metabolism to hydrocephalus in the rat. J Nutr. 1966;88(4):375-381.

23. Jones HC, Bucknall RM. Inherited prenatal hydrocephalus in the H-Tx rat: a morphological study. Neuropathol Appl Neurobiol. 1988;14(4):263-274.

24. Jimenez AR, Naz N, Miyan JA. Altered folate binding protein expression and folate delivery are associated with congenital hydrocephalus in the hydrocephalic Texas rat. J Cereb Blood Flow Metab. 2019;39(10):2061-2073.

25. Zhao R, Goldman ID. The proton-coupled folate transporter: physiological and pharmacological roles. Curr Opin Pharmacol. 2013;13(6):875-880.

26. Grapp M, et al. Molecular characterization of folate receptor 1 mutations delineates cerebral folate transport deficiency. Brain. 2012;135(Pt 7):2022-2031.

27. Steinfeld R, et al. Folate receptor alpha defect causes cerebral folate transport deficiency: a treatable neurodegenerative disorder associated with disturbed myelin metabolism. Am J Hum Genet. 2009;85(3):354-363.

28. Huemer M, Baumgartner MR. The clinical presentation of cobalamin-related disorders: From acquired deficiencies to inborn errors of absorption and intracellular pathways. JInherit Metab Dis. 2019;42(4):686-705.

29. Rossi A, Biancheri R, Tortori-Donati P. The pathogenesis of hydrocephalus in inborn errors of the single carbon transfer pathway. Neuropediatrics. 2001;32(6):335-336.

30. Narisawa A, et al. Mutations in genes encoding the glycine cleavage system predispose to neural tube defects in mice and humans. Hum Mol
Genet. 2012;21(7):1496-1503.

31. Ishida $\mathrm{M}$, et al. A targeted sequencing panel identifies rare damaging variants in multiple genes in the cranial neural tube defect, anencephaly. Clin Genet. 2018;93(4):870-879.

32. Shah RH, Northrup H, Hixson JE, Morrison AC, Au KS. Genetic association of the glycine cleavage system genes and myelomeningocele. Birth Defects Res Part A Clin Mol Teratol. 2016;106(10):847-853.

33. Chang HM, Martinez NJ, Thornton JE, Hagan JP, Nguyen KD, Gregory RI. Trim71 cooperates with microRNAs to repress Cdkn1a expression and promote embryonic stem cell proliferation. Nat Commun. 2012;3:923.

34. Kim JK, et al. Srg3, a mouse homolog of yeast SWI3, is essential for early embryogenesis and involved in brain development. Mol Cell Biol. 2001;21(22):7787-7795.

35. Harmacek L, et al. A unique missense allele of BAF155, a core BAF chromatin remodeling complex protein, causes neural tube closure defects in mice. Dev Neurobiol. 2014;74(5):483-497.

36. Chen Z, et al. Mice deficient in methylenetetrahydrofolate reductase exhibit hyperhomocysteinemia and decreased methylation capacity, with neuropathology and aortic lipid deposition. Hum Mol Genet. 2001;10(5):433-443. 